\title{
Recursos hídricos, contaminación y sociedad civil en la región occidental de Honduras
}

Jorge Humberto Orellana*

Francisco Machado Leiva* Álvaro Rivas Guzmán*

\section{RESUMEN}

El occidente hondureño es rico por su oferta ambiental, recursos hídricos, biodiversidad. Diferentes organizaciones de desarrollo están preocupadas por el manejo del recurso hídrico en la región, ejerciendo acciones de investigación e incidencia política ambiental.

El objetivo con el presente trabajo es presentar el rol fundamental de la sociedad civil para evaluar y medir indicadores químicos de calidad de agua de ríos y afluentes del río Higuito (Lara, San Andrés), así como presentar datos para generar consciencia de los posibles problemas ambientales y en la salud humana de los pobladores, y como a partir de estas evidencias científico técnicas se plantea el posible riesgo de la calidad del recurso hídrico de la principal ciudad de occidente, Santa Rosa de Copan.

Se realizó un muestreo de aguas para medir propiedades físicas y químicas en diferentes quebradas del municipio de la Unión. Se entrevisto a líderes y liderezas de las aldeas cercanas, así como a técnicos de ONGs y gobiernos locales. Se evidencia a partir del análisis químico del agua presencia de metales en tres corrientes de aguas superficiales, por encima de lo permitido por la Organización Internacional de la Salud. Además se aprecia la vulnerabilidad ambiental que presentan las cuencas por acción de la industria minera, agricultura, falta de políticas y planes de manejo ambiental. Se valora el rol fundamental en la protección de los recursos naturales de la sociedad civil organizada.

Es fundamental integrar el conocimiento científico técnico liderado por los centros de investigación o universidades con organizaciones de sociedad civil, e instancias

* Msc Jorge Humberto Orellana, jorellana4000@yahoo.es

Centro Universitario de la Región Occidental CUROC-UNAH

${ }^{*}$ Msc. Francisco Machado Leiva

Presidente Ejecutivo Asociación de Organismos no Gubernamentales

*PhD. Alvaro Rivas Guzmán, arivasgot@hotmail.com

Universidad de Göttingen, Alemania 
gubernamentales responsables de la protección ambiental y de recursos hídricos, para coordinar acciones eficaces y eficientes en la protección del recurso hídrico.

\section{Palabras Clave: Sociedad civil, Contaminación, Agua.}

\section{ABSTRACT}

The Western Honduras is rich because of the environmental offer, hidryc resources, biodiversity. Different development organization are concern about the hidryc resources management of the region, taking action in the areas of research and environmental policy incidence.

The main objective of this work its to present the main rol of the civil society in order to evaluate and measure the presence of chemical components and water quality of the rivers and river affluent (Lara and San Andres) and so to present data in order to promote conciousmess of the possible environmental problems and its impact on the human health population, and from the base of these results base on scientific evidence, determine the quality of the water of the city of Santa Rosa de Copan.

Simples were taken in order to measure the quality of the water, the simple were taken from different point of the Lara's river on the county of La Unión. Interviews were performed to community leaders and staff of NGO's working in the area.

Base on previous analysis, heavy metals were found in the water of the affluent of the Lara's river. Besides the vulnerability of the Lara river because of the mining industry, agriculture lack of policies and environmental management planning. Its important to determine the rol of the civil society in order to protect and preserve the environment.

It is fundamental to integrate the scientific knowledge, leaded by universities and research centers, civil society organizations and governmental offices responsible to protect the environment and the hidryc resources, in order to coordinated actions for the protection of this resources.

Key words: Civil society, pollution, water 


\section{INTRODUCCIÓN}

El derecho al agua está inseparablemente relacionado con otros derechos humanos, incluidos el derecho a la salud, el derecho a la vivienda y el derecho a alimentos adecuados. Por consiguiente es una responsabilidad de todos lo actores de la sociedad: Organizaciones del Estado, Organizaciones Privadas, ONG's, Organizaciones de Sociedad Civil, empresarios, centros de investigación y educación, de trabajar por el cuidado del agua como recurso público.

El occidente Hondureño es rico en su oferta ambiental específicamente en recursos hídricos beneficiando el desarrollo del país y países vecinos -El Salvador, Guatemala- pues en esta zona nacen ríos importantes como por ejemplo el Lempa y Chamelecón. Por consiguiente, cuidar, conservar y trabajar en propuestas sostenibles económicamente y ambientalmente son una urgencia y prioridad para la región. Las cuencas de la región exigen de un mejor manejo tanto en su parte técnica como político-administrativa además de contar con la participación activa de la sociedad civil en el proceso de protección, cuidado e incidencia política ambiental para los recursos hídricos que garanticen el éxito de la protección ambiental y del recurso agua.

En este sentido las principales amenazas en Honduras de sus cuencas son: Ausencia de planes de manejos; ausencia en el manejo racional de los residuos sólidos y desechos de la agroindustria, deforestación, etc.

Nuestro propósito con el presente trabajo es sensibilizar a la opinión pública y científica de la importancia en debatir sobre los problemas ambientales, específicamente los recursos hídrico, los peligros relacionados con la contaminación del agua y las estrategias de incidencia surgidas desde las necesidades propias de la población, con la firme convicción que sólo con acciones coordinadas y con el conocimiento científico pertinente, se podrá incidir ante los tomadores de decisiones para formular políticas públicas eficaces a favor de las comunidades más postergadas y excluidas del país, aprovechando además los esfuerzos que desde la sociedad civil organizada se realizan en favor de la defensa de los recursos naturales como bienes públicos en el país. Así, pues, con el apoyo coordinado de universidades, gobiernos locales, organizaciones de la sociedad civil es que se podrá proponer ante los tres poderes del Estado iniciativas de ley que protejan los recursos hídricos, forestales y ambientales de la región occidental y del país en general. 


\section{MÉTODO}

\section{Diseño}

En la presente investigación se han utilizado dos metodologías diferentes: Para abordar todo lo relacionado con la participación de los actores locales se ha requerido las técnicas metodológicas de la investigación acción participativa (IAP) Con el fin de recoger las apreciaciones y percepciones de los actores de la sociedad civil de la región respecto a sus recursos hídricos y para la presentación de indicadores químicos de calidad de suelos, se tuvo la colaboración del Centro de Investigaciones Acuáticas CIRA adscrita a la Universidad Nacional Autónoma de Nicaragua UNAN, por lo que se trabajó con sus técnicas y metodologías de laboratorio para análisis de agua.

\section{Población}

Se realizaron muestras aleatorias en los ríos Lara, Quebrada San Andrés y Casas Viejas, del municipio de la Unión Copán.

\section{Entorno}

El muestreo de agua se hizo en los ríos Lara, Casas Viejas y San Andrés del municipio de la Unión Copán que son afluentes del río Higuito que abastece de agua a la ciudad de Santa Rosa de Copán, con la participación de líderes comunitarios de las respectivas aldeas circunvecinas de estos ríos.

\section{Intervenciones}

\section{Evaluación de indicadores da calidad (cuantitativos)}

Se realizó el levantamiento de cinco muestras de agua, tomadas en diferentes puntos de una micro-cuenca denominada río Lara afluente del río Higuito. Cercanas a la explotación minera de la zona. Las muestras fueron georeferenciadas.

\section{Analizándose los siguientes parámetros de campo: pH, Conductividad eléctrica}

Para el análisis de laboratorio de metales pesados: Aluminio total, Arsénico, total, Cadmio total, Cinc total, Cobre total, Cromo total, Manganeso total, Mercurio total, Níquel total, Plomo total, Selenio total, los análisis han sido efectuados con un espectrofotómetro UV/VIS que es una técnica que permite una alta precisión en los análisis del agua.

\section{Observaciones Directas}

Se constató a través de la observación directa que en zonas de vocación forestal altamente erosionada y eliminación de la capa orgánica del suelo producto de la 
explotación minera. Algunas comunidades como San Miguel en la Unión Copán, dista a sólo 300 metros de distancia de las pilas de lixiviación de la industria minera.

\section{Análisis de muestras}

Los análisis han sido efectuados con un espectrofotómetro UVIVIS que es una técnica que permite una alta precisión en los análisis del agua, haciéndose las respectivas comparaciones de las muestras recolectadas con los niveles permitidos por la Organización Mundial de la Salud OMS. Se recogió además la opinión a través de la entrevista con los pobladores sobre la percepción que tienen sobre el tema de la minería y los problemas de contaminación de los afluentes de agua.

\section{RESULTADOS}

\section{Análisis y contexto: El Recurso hídrico}

Indudablemente, el mundo enfrenta una crisis cada vez más preocupante con el tema del agua, que va desde sequías en algunas partes del mundo, desperdicio del vital liquido en otras regiones producto de la agricultura extensiva, hasta problemas relacionados con la demografía, el calentamiento global y la contaminación por las industrias de producción y de extracción de metales. En su libro "La tierra explota, superpoblación y desarrollo" Giovanni Sartori, nos recuerda El agua es cada vez más el bien primario que de manera más clara separa el Norte del Sur del Mundo: por lo que los debates en este sentido son que en un futuro próximo las guerras sean por el abastecimiento y el control del recursos hídricos, hasta la fecha son simples debates sin embargo ya hay países y Estados en el mundo que defienden el derecho por la soberanía de ríos que bañan sus fronteras. Hablar de agua en los países en desarrollo significa dirigir la mirada a una serie de problemas que tienen que ver con la subsistencia en sus habitantes, con educación, con salud y con contaminación para citar algunos ejemplos.

El agua como recurso estratégico para una Nación y específicamente para el Occidente de Honduras, es un tema que debe estar en la agenda de las organizaciones de la sociedad civil, debido al riesgo de explotación de este recursos que presenta la globalización materializados en los Tratados de Libre Comercio y el Plan Puebla Panamá (PPP), pues para los intereses geopolíticos de algunos países, el agua representa uno de los recursos de mayor importancia.

Hay que tener en cuenta que en Centroamérica hay aproximadamente 120 cuencas hidrográficas principales, de las cuales 23 son internacionales; éstas significan el $19 \%$ de los sistemas hídricos de la región y el $10.7 \%$ de las cuencas internacionales del planeta. (Ramos 2004) Por dicha razón algunas iniciativas como el Plan Puebla 
Panamá, pretende apropiarse del recurso hídrico a través de represas hidroeléctricas y un acueducto continental que vienen desde Colombia, pasando por Centroamérica ciudad de México hasta la zona sur de USA. De allí el reto de la saciedad civil, las universidades y las comunidades locales para que se organicen e incidan ante sus gobiernos locales y nacionales para defender la soberanía del agua en Mesoamérica. En Honduras, El Salvador, Guatemala y Nicaragua hay proyectos en ejecución para la construcción de represas hidroeléctricas:

Por ejemplo la represa El Tigre según los debates que ha habido al respecto, toda el agua que generaría esta represa sería cedida a EI Salvador para su administración y posterior venta a Honduras, lo que resultaría una mala negociación del recurso hídrico y de la propia soberanía del país.

Uno de los temas centrales que ha venido desarrollando la sociedad civil en el Occidente hondureño a través de la Alianza Cívica por la Democracia ACD, es la defensa y protección de los recurso hídricos como consecuencia del impacto ambiental generado por la industria extractiva de la minería en la region. En Honduras hay más de 372 concesiones de exploración y 57 de explotación minera. A nivel nacional existen un total de 335.359 kilómetros cuadrados en poder de las mineras, prácticamente el $30 \%$ del territorio nacional.

La Ley General de Minería de Honduras en 1998 (Decreto legislativo 292 de 1998) ha facilitado el otorgamiento de licencias y concesiones mineras que les confiere derechos de explotación a estas empresas en gran parte del territorio hondureño sin considerar: la participación local, la protección ambiental de cuencas, poblados, pagos de impuestos muy bajos ( $1 \%$ de impuestos al municipios), débiles derechos laborales y ninguna consideración para la salud de pobladores.

La Industria minera ha aumentado en la región del occidente hondureño, existiendo varias concesiones para la explotación. Los riesgos de la actividad minera son: Contaminación de las cuencas hídricas con cianuro y metales pesados; disminución del caudal de aguas, a largo plazo contaminan con el residuo ácido o básico de minas que es el desprendimiento de metales pesados de las rocas y se depositan progresivamente en las fuentes de agua, representando eminentemente un peligro para los consumidores del agua en el occidente de Honduras.

\section{Riesgos en el consumo del Agua el caso de Santa Rosa de Copán}

La ciudad de Santa Rosa de Copán, que es la principal cabecera municipal de Occidente y que alberga más de 50.000 habitantes, se encuentra en una zona de riesgo por contaminación de sus afluentes hídricos para el consumo humano por acción de la industria minera, ya que a unos pocos kilómetros de esta ciudad en el 
municipio de la Unión Copán, se encuentra una de las empresas mineras que operan en el país desde hace varios años. Realizando la extracción de oro a cielo abierto con utilización de cianuro, representando un peligro por aguas residuales del proceso de extracción del oro, que pueden fácilmente contaminar los afluentes del Río Higuito que abastece de agua para consumo a la ciudad de Santa Rosa de Copán, considerando además que este afluente surte también de agua a otros municipios por donde atraviesa su cauce del río.

Se estima que en el 2003 se vertieron 300 galones de cianuro al río Lara lo que produjo la muerte de más de 18.000 peces y en junio del 2006 descargaron las pilas No.2 y 3 sin cumplir con los requisitos administrativos ante la Dirección de Fomento a la Minería (DEFOMIN), y sin informar a las entidades gubernamentales respectivas (ASONOG 2007). Esto evidencia la situación de riesgo de la ciudad de Santa Rosa de Copán, frente al consumo de agua contaminada. Es por ello que actualmente se realizan acciones de desarrollo, investigación e incidencia en temas de gobernabilidad del recurso hídrico, manejo de cuencas, pago por servicios ambientales, contaminación química, manejo de aguas servidas, ley de agua, ordenamiento territorial entre otras.

La Ley general del Ambiente 1993 plantea la importancia del ordenamiento territorial como una estrategia técnica para el manejo adecuado, sostenible y con criterio ambiental de las cuencas, infortunadamente algunas instituciones municipales de la región carecen de información técnica y voluntad política para el ejercicio de dichas actividades.

\section{Estado del Problema Local}

Santa Rosa de Copán y los municipios contiguos: Talgua Lempira, Cucuyagua y Corquín Copán, Belén Gualcho, Sensenti, La Labor, San Marcos y Lucerna Ocotepeque, forman parte de la microcuenca del río Higuito, muchos de estos municipios se abastecen de esta agua superficial, o drenan sus aguas residuales al mismo. De ahí que las principales amenazas de esta microcuenca son:

1. Vertimiento de sustancias químicas por la industria minera.

2. Vertimiento de aguas negras de los municipios de la parte media dela cuenca.

3. Ausencia de planes de manejo ambiental.

4. Ausencia de un monitoreo de alerta temprana para la evaluación periódica de metales pesados.

5. Deforestación

Esto representa un reto para diferentes sectores no sólo de las organizaciones 
ambientales de la sociedad civil, sino la voluntad política de los gobiernos locales, la cooperación internacional y sobre todo las universidades que tienen presencia en esta región.

\section{Participación de la Sociedad Civil y Organizaciones no Gubernamentales en procesos de incidencia ambiental}

La gestión de riesgo es la formulación y la implantación de medidas y procedimientos técnicos y administrativos orientados a prevenir, controlar o reducir los riesgos existentes. Dentro de los instrumentos de gestión ambiental se encuentran los Programas de vigilancia ambiental, que consisten en el seguimiento sistemático de la variación temporal y espacial de varios parámetros ambientales referidos a la calidad de los suelos, agua, etc. La participación ciudadana en la gestión ambiental es un derecho y debe entenderse como el conjunto de acciones que pueden realizar los actores sociales involucrados para atender sus preocupaciones ambientales. La comunidad local es un actor principal con intereses legítimos sobre su hábitat y medio ambiente. Como actor activo no sólo tiene el interés sino el derecho y la responsabilidad de tomar acciones para prevenir daños ocasionados por los fenómenos naturales, las actividades industriales y su propia actividad cotidiana. (Martines Castillo Z, 2003).

En este sentido consideramos que uno de los retos fundamentales que tienen las organizaciones de desarrollo y la sociedad civil en el occidente de Honduras, es velar porque sus recursos hídricos sean manejados con la responsabilidad ambiental que merecen todos sus habitantes, a tal efecto la participación de las organizaciones puede especificarse en dos niveles:

1. Involucrando a técnicos de las ONGs, Juntas de Agua, Unidades Municipales Ambientales UMAS correspondiente a cada municipio y así adquieran destrezas técnicas para la toma de muestras de agua y evaluación de los niveles permisibles de los metales pesados en el agua de consumo.

2. Las organizaciones de desarrollo aglutinadas en redes, y otras entidades de sociedad civil como son patronatos, organizaciones gremiales campesinas, indígenas, cooperativas, iglesias, han logrado constituir Alianzas como instancias que tiene el poder movilizador de la sociedad civil para reivindicar la protección ambiental de las cuencas hidrográficas, como consecuencia de las malas políticas ambientales públicas específicamente las Leyes de minería, forestal y recursos hídricos, por lo que su labor de incidencia, movilización, reclamo y sensibilización es fundamental para la protección de las fuentes de agua y el ambiente en general. 
A nivel regional y nacional, ha habido un proceso participativo de parte de estas instituciones para conocer las diferentes leyes relacionadas con recursos naturales, además de elaborar una estrategia de incidencia política para proponer y exigir ante las instancias gubernamentales respectivas una nueva política ambiental referente al manejo de la minería, manejo del bosque y agua. Con el fin de realizar incidencia política ambiental toda la información técnico-científico de indicadores de metales pesados solo tiene valides jurídica en la medida que en la toma de muestras se involucren figuras institucionales públicas como Derechos Humanos, Fiscalía del Ambiente, y los esfuerzos que desde sociedad civil se hacen por la transparencia y el combate a la corrupción, a través de los comisionados municipales y las comisiones ciudadanas de transparencia de cada municipio.

\section{Parámetros químicos de las muestras de agua}

La Norma Técnica para la Calidad del Agua Potable, en su Título III Especificaciones Técnicas, Artículo 3, que reza "esta norma de calidad de agua establece los requisitos básicos a los cuales debe responder la calidad del agua suministrada en los servicios para consumo humano y para todo uso doméstico, independientemente de su estado, origen, o grado de tratamiento". Por lo tanto, los Resultados de los Análisis de Laboratorio fueron evaluados conforme al Acuerdo 084 de esta Norma y a continuación presentamos los parámetros encontrados y sus concentraciones máximas permisibles. En la Tabla 1 se muestran los valores de metales pesados de mayo del 2006 de Manganeso, Plomo, Arsénico y se aprecia como superan el valor permitido, según Acuerdos 084.

\section{Tabla 1}

\section{Concentraciones de Metales pesado en las muestras Mayo 2006}

\begin{tabular}{|l|c|c|c|c|}
\hline \multicolumn{1}{|c|}{$\begin{array}{l}\text { Identificación } \\
\text { Muestra }\end{array}$} & $\begin{array}{c}\text { Metales } \\
\text { encontrados }\end{array}$ & $\begin{array}{c}\text { Concentración } \\
\text { máxima } \\
\text { permitida } \\
\text { según } \\
\text { Acuerdo 084 }\end{array}$ & $\begin{array}{c}\text { Concentración } \\
\boldsymbol{\mu g} / \mathrm{l}\end{array}$ & $\begin{array}{c}\text { Concentración } \\
\mathbf{M g} / \mathrm{l}\end{array}$ \\
\hline $\begin{array}{l}\text { Quebrada San } \\
\text { Andrés }\end{array}$ & Manganeso & $0.01 \mathrm{mg} / /^{*}$ & 99.00 & 0.099 \\
\cline { 2 - 5 } & Plomo & $0.01 \mathrm{mg} / \mathrm{l}$ & 13.77 & 0.0137 \\
\hline $\begin{array}{l}\text { Quebrada Casa } \\
\text { Vieja }\end{array}$ & Manganeso & $0.01 \mathrm{mg} / \mathrm{l}$ & 15.75 & 0.0157 \\
\hline $\begin{array}{l}\text { Río Lara Aguas } \\
\text { Abajo }\end{array}$ & Manganeso & $0.01 \mathrm{mg} / /^{*}$ & 67.00 & 0.067 \\
\hline Río Lara (CPD) & Manganeso & $0.01 \mathrm{mg} / /^{*}$ & 21.00 & 0.047 \\
\hline
\end{tabular}

Fuente: Centro Humboldt Nicaragua-ASONOG.

Nota: * microgramo por litro $(\mu \mathrm{g} / \mathrm{l})$ se divide entre 1000 , para obtener miligramos por litros (mg/l). 
Quebrada San Andrés: el manganeso presenta concentraciones de $0.09 \mathrm{mg} / \mathrm{l}$, por encima del valor recomendado que es de $0.01 \mathrm{mg} / \mathrm{l}$, el plomo con concentraciones de $0.0137 \mathrm{mg} / \mathrm{l}$ y el arsénico con concentraciones de $0.015 \mathrm{mg} / \mathrm{l}$ se encuentran por encima del valor máximo admisible que es de $0.01 \mathrm{mg} / \mathrm{l}$ para ambos metales.

Quebrada Casas Viejas: Se encuentra el manganeso por encima del valor recomendado que es de $0.01 \mathrm{mg} / \mathrm{l}$, dado que presenta concentraciones de 0.067 $\mathrm{mg} / \mathrm{l}$.

Río Lara: Tanto en la parte media como baja el manganeso presenta concentraciones por encima de lo permitido de $0.02 \mathrm{mg} / \mathrm{l}$ y $0.0046 \mathrm{mg} / \mathrm{l}$ respectivamente.

En otro estudio realizado en febrero del 2007, con tres puntos de muestreo, arrojaron los datos concentraciones de metales pesados en el agua muy altos de lo permitido y se consignan en la Tabla No. 2. Para el río Lara el Aluminio es de 0.12 $\mathrm{mg} / \mathrm{l}$; Cobre $3.2 \mathrm{mg} / \mathrm{l}$ y Hierro $22.6 \mathrm{mg} / \mathrm{l}$. Para el río Casas Viejas el Cobre es de 3.42 $\mathrm{mg} /$; ; Hierro $29.1 \mathrm{mg} / \mathrm{l}$ y Manganeso $1.3 \mathrm{mg} / \mathrm{l}$. Para la Quebrada San Andrés el Aluminio es de 0,81 mg/l; Cobre 2,97 mg/l y Hierro 30,2 mg/l.

Tabla No. 2

Concentraciones de Metales pesado en las muestras de febrero 2007

\begin{tabular}{|l|c|c|c|}
\hline \multicolumn{1}{|c|}{ Valor/Lugar } & $\begin{array}{c}\text { Río Lara } \\
\text { San Miguel }\end{array}$ & $\begin{array}{c}\text { Río Lara } \\
\text { San Miguel }\end{array}$ & $\begin{array}{c}\text { Quebrada } \\
\text { San Andrés }\end{array}$ \\
\hline $\mathrm{PH}$ & 8.60 & 8.80 & 7.60 \\
\hline Conductividad* $\mu \mathrm{s} / \mathrm{cm}$ & 180 & 4.40 & 700 \\
\hline TDS mg/l & 90 & 220 & 350 \\
\hline Aluminio total $\mathrm{mg} / \mathrm{l}$ & 0.12 & 0.02 & 0.81 \\
\hline Cobre total $\mathrm{mg} / \mathrm{l}$ & 3.2 & 3.42 & 2.97 \\
\hline Hierro total $\mathrm{mg} / \mathrm{l}$ & 22.6 & 29.1 & 30.2 \\
\hline Manganeso total $\mathrm{mg} / \mathrm{l}$ & 0.04 & 1.3 & 0.19 \\
\hline Zinc total $\mathrm{mg} / \mathrm{l}$ & $>0.01$ & 0.11 & $>0.01$ \\
\hline
\end{tabular}

Fuente: Flaviano Bianchini- ASONOG 2007

Resultados obtenidos como producto de la movilización de sociedad civil.

La información técnica obtenida a partir de la investigación de la calidad química del agua en occidente, permite sustentar y soportar científicamente las denuncias realizadas por organizaciones como la Alianza Cívica por la Democracia en defensa del interés público y ambiental; esto ha permitido realizar acciones de incidencia 
política a nivel de movilización y sensibilización de la población, así como otras acciones:

1. Tres movilizaciones masivas con comunidades locales para exigir al gobierno la derogación de la actual Ley de Minería.

2. Creación de mesas de discusión para formular y proponer iniciativas de políticas públicas relacionadas con la Ley Forestal, Ley de Minería, Ley de Agua, etc.

3. Presentación de una propuesta de nueva ley de Minería el pasado 18 octubre del 2006.

4. Tres reuniones con el Ejecutivo o sus representantes.

5. Dos reuniones con el Congreso Nacional de la República 2006-2007

6. Participar con aportes técnicos y políticos ante la Secretaría de Recursos Naturales para un nuevo borrador de ley de minería.

7. Diseño interno de una estrategia de Incidencia Política para cambiar políticas públicas que defiendan los recursos naturales y el interés público.

8. Sensibilización en la opinión pública nacional a través de los medios de comunicación de la problemática ambiental generada por la industria minera para las cuencas hidrográficas.

Sin lugar a dudas, es fundamental resaltar el papel que juegan las organizaciones de sociedad civil en la defensa del interés público para la protección y buen manejo de nuestros recursos hídricos, por lo tanto, las instancias de base formadas en cada uno de los municipios deben cumplir con su labor de vigilar estos recursos que son patrimonio exclusivo de sus habitantes. Organizaciones como las Juntas de Agua deben ir mas allá de su papel administrador del agua en las comunidades, enfocando su trabajo también hacia la adquisición de las destrezas técnicas para continuar con la labor periódica de vigilar la calidad del agua que consumen sus comunidades.

Consideramos que la academia debe jugar un papel protagónico en la defensa de nuestros recursos, aportando sus conocimientos técnicos y científicos en la búsqueda de la calidad del agua, la protección de los recursos, prevenir la contaminación y sobre todo de velar por mejorar las condiciones de vida de los habitantes de la región occidental y del país en general.

\section{DISCUSIÓN}

En la medida que nos acercamos a la realidad socioeconómica, cultural, política y ambiental de nuestras comunidades, nos damos cuenta del enorme potencial que existe en la región occidental en cuando a sus oportunidades de crecimiento y 
desarrollo, pero también nos damos cuenta de los peligros ambientales que la zona es sometida producto de las decisiones gubernamentales, sobre todo con los recursos naturales y especialmente con el consumo del agua de sus habitantes.

Es indudable el nivel de apropiamiento que están adquiriendo organizaciones de la sociedad civil para luchar por los recursos naturales del país, y sobre todo del agua, que representa la salud y la vida para miles de personas del occidente del país, si bien es cierto, existen los peligros inminentes de contaminación se hace necesario los diagnósticos y estudios científicos para vigilar constantemente los niveles de contaminación que pudieran provocar industrias como la extracción minera, así mismo valorar los beneficios que la misma trae consigo al país con los peligros que puede representar para las comunidades más pobres de la región. Este fenómeno deber permitir un mejor acercamiento entre la academia y las organizaciones de sociedad civil para que juntos se puedan proponer las estrategias de incidencia política para que los tomadores de decisiones legislen para el bien común y no de ciertas clases con privilegios especiales en el país. Según las muestras del análisis de agua, existe una notoria presencia de metales pesados, por encima de los valores permisibles a nivel internacional, lo que debe preocupar a ciudades como Santa Rosa de Copán, donde el consumo de agua proviene de afluentes cercanas a la industria minera.Consideramos que por la falta del equipo técnico adecuado para el análisis periódico del agua, tenemos que acudir a otras instituciones de países vecinos, pero es imperativo que estos estudios se realicen en instituciones como la Universidad Nacional Autónoma de Honduras, y con ello se realicen las pruebas periódicas y sistemáticas para la vigilancia de la calidad del agua en la región.

\section{CONCLUSIONES}

Se ha llegado a evidenciar la descarga de aguas residuales por parte de la industria minera a las aguas del Río Lara, afluente del Río Higuito, principal abastecedor de agua potable de la ciudad de Santa Rosa de Copán, lo que representa un grave peligro en el corto y mediano plazo en la salud de sus habitantes. Este afluente también es aprovechado por otros municipios aledaños.

Los resultados de la quebrada de San Andrés, demuestran presencia de tres de los once metales analizados, por encima de los valores permisibles, esto se debe al material estéril de las pilas de lixiviación, los cuales se han lixiviado hacia las aguas superficiales y muy probable a las subterráneas, debido a la textura franco arenosa del suelo y franco arcillo arenosa del subsuelo y a la pendiente de los terrenos de este municipio. 
Los dos estudios químicos realizados en diferentes fechas coinciden en que hay niveles más altos de lo permitido de Aluminio, Cobre, Hierro, manganeso en tres de los principales afluentes cercanos a la industria minera (Río Lara, Casas Viejas y SanAndrés).

En nuestras observaciones directas se puede apreciar un cambio drástico del paisaje y del deterioro de los bosques, fuentes de agua y suelo, así como el desplazamiento que han tenido comunidades como San Andrés en el municipio de la Unión Copán, lo cual ha representado una pérdida en la cultura y costumbres de sus habitantes.

Existe una notable preocupación por parte de los pobladores de la región, así como de las organizaciones de la sociedad civil por el medio ambiente en la región, y por lo tanto esto representa una oportunidad para que la academia y el colectivo de universidades puedan involucrarse a través de sus conocimientos técnicos y científicos en la problemática ambiental de la región y sobre todo del recurso hídrico, ya que occidente es considerada una de las regiones que más produce agua, la cual abastece lógicamente al resto del país a través de sus ríos.

El movimiento social en Honduras y sobre todo en la región occidental ha logrado una forma alternativa para exigir reivindicaciones ambientales, sociales y públicas conservando la independencia de partidos políticos tradicionales. Pero estas organizaciones deben tener planes estratégicos que les permita ser eficientes en sus luchas para la obtención de mejores resultados.

\section{AGRADECIMIENTOS}

Quisiéramos dar nuestro agradecimiento a las comunidades de San Miguel y San Andrés en la Unión Copán, al presbiterio de occidente por su fuerte lucha por la defensa y ética de la vida, al Instituto Humboldt donde se realizaron los análisis de las muestras recolectadas, a la Asociación de Organismos no Gubernamentales por el apoyo logístico y financiero para estos estudios así como a la cooperación solidaria internacional.

\section{BIBLIOGRAFÍA}

Caballero Zeitun E. Madrid Rossell Z, Valladares L, Castilla J, Carias P, Corrales R, Najarro, T, et.al. El occidente de Honduras, tierra de carencias y esperanzas: tendencias de desarrollo e impactos de los procesos de intervención pública en los últimos 50 años. 1 ed., Tegucigalpa: Alin; 2005. 
Lovelock J, La venganza de la Tierra. 1 ed., Barcelona: Planeta; 2007.

Martínez Castillo Z. Guías prácticas para situaciones específicas: manejo de riesgos y preparación para respuestas a emergencias mineras. 1 ed. Santiago de Chile. CEPAL División de Recursos Naturales e Infraestructura. 2003.

Sartori, G. Mazzoleni, G. La Tierra Explota, Superpoblación y Desarrollo. 1 ed., Madrid:Gráficos de Rogar, S.A.; 2003.

\section{Estudios Técnicos}

Bianchini Flaviano. Estudio Técnico Calidad de agua en el área de explotación minera del proyecto San Andrés (municipio de la Unión, departamento de Copan). Santa Rosa Copán. 2007

Machado, F, Cárcamo, C. Las industrias extractivas: Minería, un mal negocio para Honduras. ASONOG. Oxfam, Christian Aid, 2005.138p.

Moran Robert E., Cual es el gran secreto, observaciones y comentarios acerca de la Mina de SanAndrés. Santa Rosa Copán: Dan Church Aid. 2005.

Moran, Robert E, Cyanide in Mining: Some Observations on the Chemistry, Toxicity and Analysis of Mining-Related Waters. En: Proc. Central Asia Ecology99, Lake Issyk Kul, Kyrgyzstan. June 1999.

Ortiz Felipe. Estudio Técnico. Santa Rosa Copán: Centro Humboldt; 2006.

Ramos Delgado G.C. Privatización y saqueo del agua en Mesoamérica. Revista Ecoportal. 2005 